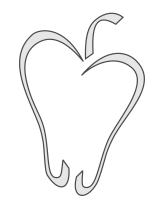

ISSN 1852-4249

\title{
Dilute antiferromagnetism in magnetically doped phosphorene
}

\begin{abstract}
A. Allerdt, ${ }^{1}$ A. E. Feiguin ${ }^{1 *}$
We study the competition between Kondo physics and indirect exchange on monolayer black phosphorous using a realistic description of the band structure in combination with the density matrix renormalization group (DMRG) method. The Hamiltonian is reduced to a one-dimensional problem via an exact canonical transformation that makes it amenable to DMRG calculations, yielding exact results that fully incorporate the many-body physics. We find that a perturbative description of the problem is not appropriate and cannot account for the slow decay of the correlations and the complete lack of ferromagnetism. In addition, at some particular distances, the impurities decouple forming their own independent Kondo states. This can be predicted from the nodes of the Lindhard function. Our results indicate a possible route toward realizing dilute anti-ferromagnetism in phosphorene.
\end{abstract}

\section{Introduction}

Phosphorene, a single layer of black phosphorus, is one of the many allotropes of the element. Others include red, white, and velvet phosphorus. Of these, black is the most thermodynamically stable and least reactive [1]. Its crystalline structure resembles that of graphite, whose 2D form is famously known as graphene, and can similarly be fabricated into $2 \mathrm{D}$ layers. The main qualitative distinction lies in the fact that phosphorene has a "puckered" hexagonal structure which is responsible for opening a gap in the band structure. In addition, the bonding structure is vastly different, since phosphorene does not have $s p^{2}$ bonds, but is composed of $3 p$ orbitals $[2,3]$.

Successful production of monolayer black phosphorus has been achieved only in the last few years, with the first publications concerning its single layer properties appearing in 2014 [4-7]. While being a semi-conductor with a small direct band gap in bulk form $(\sim 0.3 \mathrm{eV})$ [8], the gap increases as the number of layers is de-

\footnotetext{
*a.feiguin@northeastern.edu

1 Department of Physics, Northeastern University, Boston, Massachusetts MA 02115, USA.
}

creased.

Since its appearance, there have been many proposed promising applications and exotic properties. Besides being a semi-conductor with a band gap in the optical range, it has ample flexibility and high carrier mobility $[4,9]$. Further, it also possesses interesting optical properties such as absorbing light only polarized in the armchair direction, indicating a possible future as a linear polarizer [10]. Its stable excitons present possible applications in optically driven quantum computing [11]. Interest on its applications continues to grow. For a comprehensive review, we refer to Ref. [11].

Our understanding of magnetic doping in phosphorene is still in its infancy. A thorough study of metal adatoms adsorbed on phosphorene was performed in Ref. [12] where a variety of structural, electronic and magnetic properties emerge. The authors show the binding energies are twice the amount in graphene. A DFT study has proposed possible chemical doping by means of adsorption of different atoms, ranging from $n$-type to $p$-type, as well as transition metals with finite magnetic moment [13]. Another conceivable method of moving the Fermi level is to induce strain on the lattice, causing the level to move into the conduction band [14]. The case of magnetic impurities is considerably 




Figure 1: Phosphorene lattice showing the directions chosen to place the impurities for calculations shown in Figs. 3, 4, and 5. The numbering of lattice sites refers to the impurity separations in the same figures. Also shown are the five hopping parameters borrowed from Ref. [5]. The labels A, B, C and D refer to the four sublattices.

non-trivial, since one has to account for the Kondo effect [15] with the impurity being screened by the conduction spins in the metallic substrate, and the RKKY interaction [16-18], an effective indirect exchange between impurities mediated by the conduction electrons. These two phenomena are expected to be present and compete in phosphorene when the Fermi level is not sitting in the gap. The RKKY interaction in phosphorene with the inclusion of mechanical strain is investigated in Ref. [14]. However, all their calculations are based on second order perturbation theory and ignore the effects that Kondo physics can induce. In this work, we present numerical results for two Kondo impurities in phosphorene that capture the full many-body physics and we discuss the competition between Kondo and indirect exchange.

\section{Model and numerical method}

The Hamiltonian studied in this work is the twoimpurity Kondo model, generically written as:

$$
H=H_{\text {band }}+J_{K}\left(\vec{S}_{1} \cdot \vec{s}_{\mathbf{r}_{1}}+\vec{S}_{2} \cdot \vec{s}_{\mathbf{r}_{2}}\right),
$$

where $H_{\text {band }}$ is the non-interacting part describing the band structure of the material while the impurities are locally coupled to the substrate at positions $\mathbf{r}_{1}$ and $\mathbf{r}_{2}$ via an interaction $J_{K}$. Here, $\vec{S}_{i}$ represent the impurities, and $\vec{s}_{\mathbf{r}_{i}}$ is the spin of the conduction electrons at site $\mathbf{r}_{i}$. This problem has been theoretically studied in other

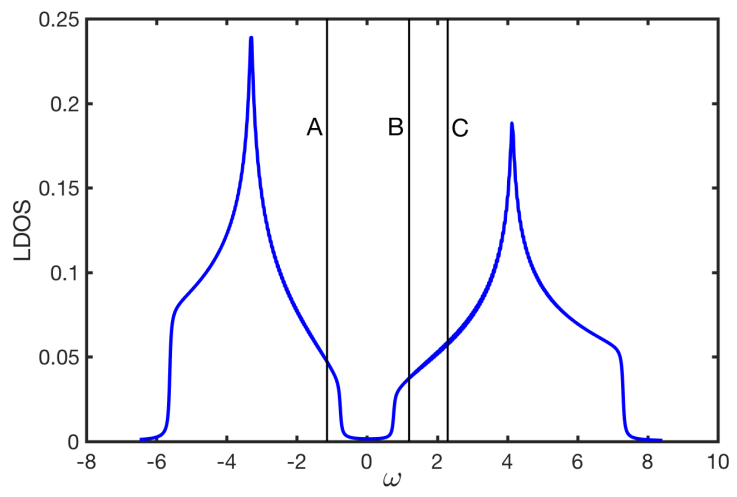

Figure 2: Local density of states of phosphorene. Vertical lines show positions of the different Fermi energies used in the calculations.

materials using a variety of approaches. This work, however, will take a non-perturbative approach revealing important subtle points. Traditionally, the RKKY interaction is characterized by the Lindhard function in second order perturbation theory, which takes the form:

$$
\chi_{i j}=2 \Re\left[\sum_{E_{\alpha}>E_{f}>E_{\beta}} \frac{\langle i \mid \alpha\rangle\langle\alpha \mid j\rangle\langle j \mid \beta\rangle\langle\beta \mid i\rangle}{E_{\alpha}-E_{\beta}}\right],
$$

where $|i, j\rangle$ represent lattice sites and $|\alpha, \beta\rangle$ are eigenstates of $H_{\text {band }}$. This function oscillates and changes sign with a period that depends on the density of the electrons. When the full geometry of the problem is taken into account, the picture becomes more complex, since a system could have a complex Fermi surface even with more than one band crossing the Fermi level in some cases [19]. However, it has been shown by the authors in previous studies [19-21] that this does not uncover the full picture.

We adapt the four-band model introduced in Ref. [5] to obtain a tight-binding description of the phosphorene band structure. Five hopping parameters are employed to closely reproduce the bands near the Fermi level with a gap of approximately $1.6 \mathrm{eV}$. These are shown schematically in Fig. 1, and their values are restated in Table 1. A plot of the density of states is shown in Fig. 2. All energies in the calculations are in units of $\mathrm{eV}$. To see the band dispersions, we refer to Ref. [5].

In order to solve the interacting problem we first perform a unitary transformation to map the quadratic part of the Hamiltonian $H_{\text {band }}$ onto an equivalent onedimensional one, as described in detail in Refs. [20,22]. 


\begin{tabular}{|c|c|}
\hline$t_{1}$ & -1.220 \\
\hline$t_{2}$ & 3.665 \\
\hline$t_{3}$ & -0.205 \\
\hline$t_{4}$ & -0.105 \\
\hline$t_{5}$ & -0.055 \\
\hline
\end{tabular}

Table 1: Tight binding hopping parameters in eV used in the calculations throughout this work.

The full many-body calculation can in turn be carried out using the density matrix renormalization group (DMRG) algorithm [23-25] with high accuracy and without approximations. For all DMRG calculations, the total system size is $L=124$ (including impurities), and we fix the truncation error to be smaller than $10^{-7}$. It is known that phosphorene nano-ribbons can host quasi-flat edge states whose emergence is topologically similar to graphene [26]. A study of their even/odd properties under application of an electric field has recently been performed [27], revealing a gap opening in these edge states. Edge states can introduce notable finite-size effects [21]. However, due to the geometry of the lattices produced by the Lanczos transformation employed in this work, these edge states are not present. In addition, we shall focus our study in a regime away from half-filling.

\section{Results}

We start by calculating the Lindhard function as a reference using Eq. (2). Figure 3 shows results for two different Fermi energies, one in the conduction band, and one in the valence band. Notice the different period of oscillation, which is to be expected from conventional RKKY theory [16-18]. We also show, in different panels, the DMRG result for the impurity spin-spin correlations $\left\langle S_{1}^{z} S_{2}^{z}\right\rangle$. Since the system is $S U(2)$ symmetric, only the $z$-component is displayed. For small values of $J_{K}$ we find that he RKKY correlations saturate at $-1 / 4$. This is an indication that the two impurities are decoupled forming "free moments". When this happens, the spins can be pointing in either direction yielding a four-fold degenerate ground state. Since we force $S^{z}=0$ in our calculations, the impurities have no choice but to align anti-ferromagnetically. This is a finite size effect that is expected when the interaction $J_{K}$ is of the order of the level spacing in the non-interacting bands. Apart from short distances, the spin correlations roughly follow the oscillation patterns predicted

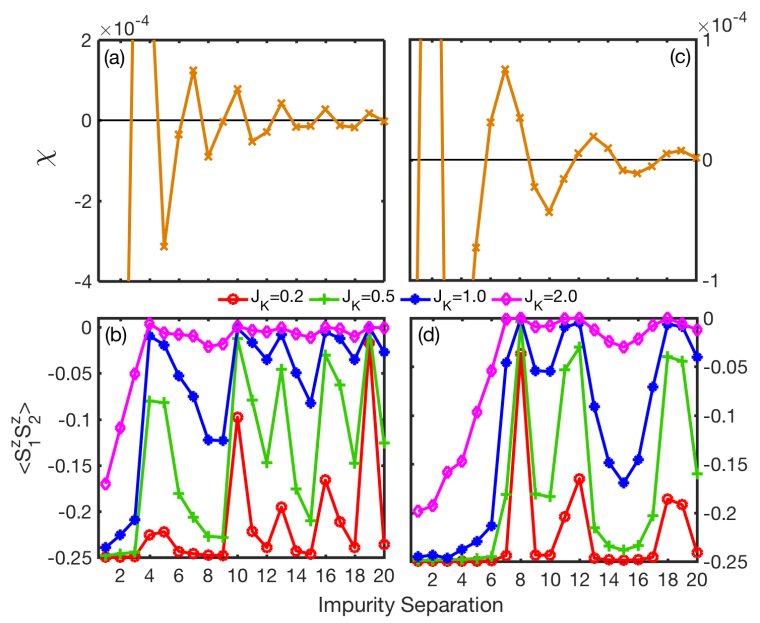

Figure 3: (a) Lindhard function and (b) spin-spin correlations along the zig-zag direction with the Fermi level at position A in Fig. 2. (c) and (d) are the same quantities with the Fermi level at position $\mathrm{C}$.

by the Lindhard function with one striking difference: ferromagnetism is non-existent. This lack of ferromagnetism is also seen in previous works [19-21]. However, in doped graphene, for example [21], the correlations depart drastically from the Lindhard function, and Kondo physics dominates after just a few lattice spacings. Here, we find that RKKY physics plays the dominant role, with correlations persisting even for quite large coupling $\left(J_{K}=2.0 \mathrm{eV}\right)$, which is greater than the band gap.

Figure 4 again the Lindhard function and spin correlations, but for a different Fermi energy and in an extended range. This is to highlight the fact that the RKKY oscillations survive at large distances with little signs of decay. Ferromagnetism is again absent in contrast with the predictions of perturbation theory, exemplifying the need for exact numerical techniques that can capture the many-body physics. The impurities, however, do appear to form their independent Kondo singlets at some particular distances. This results in the impurities being completely uncorrelated $\left\langle S_{1}^{z} S_{2}^{z}\right\rangle=0$ and typically occurs at positions where the Lindhard function has a node, as observed in Ref. [20].

To show that these effects are not due to the particular directions chosen, calculations were done along other paths with qualitatively similar results. As an example, we show in Fig. 5 the correlations along the perpendicular direction with the Fermi level at posi- 


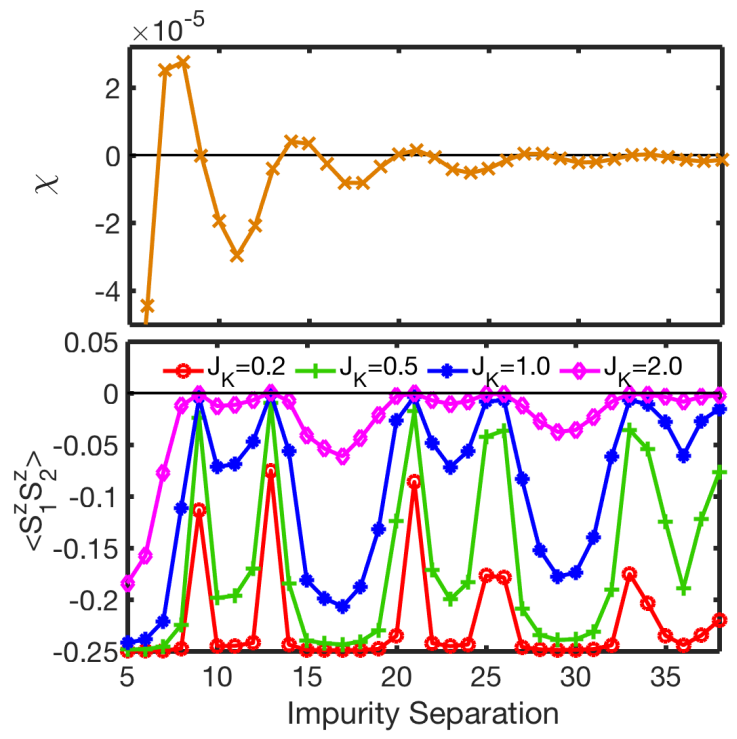

Figure 4: Lindhard function (top) and spin-spin correlations (bottom) along the zig-zag direction with the Fermi level at position B in Fig. 2. Results are shown with an extended range, highlighting the persistent oscillations even for large $J_{K}$.

tion B. As can be seen from the lattice structure, single layer phosphorene contains four sublattices, as labeled in Fig. 1. Assuming the first impurity is placed on an ' $\mathrm{A}$ ' site, along the zig-zag direction the impurities follow an " $A-A, A-B, A-A, A-B \ldots$.." pattern. Alternatively, the second impurity could be placed on the other "layer" along the zig-zag direction resulting in a " $A-C, A-D, A-C, A-D \ldots$.." pattern. It is clear that $A-D$ is equivalent to $B-C$. The armchair direction, as it has been defined, consists of impurities always on the same sublattice. In all cases (not shown), ferromagnetism is absent and we find dominant antiferromagnetic RKKY interactions.

\section{Conclusions}

By means of a canonical transformation and exact numerical calculations using the DMRG method, we have studied the competition between Kondo and RKKY physics on phosphorene. The method is numerically exact, and even though it is limited to finite systems, these can be very large, of the order of a hundred lattice sites and more. Our results highlight the non-perturbative nature of the RKKY interaction and the non-trivial ab-


Figure 5: Lindhard function (top) and spin-spin correlations (bottom) along the armchair direction with the Fermi level at position B in Fig. 2.

sence of ferromagnetism. This remains an outstanding question and should stimulate more research in this direction. It is possible that by adding a repulsive interaction between conduction electrons, the system may acquire a net magnetic moment, a behavior of this type has been observed in graphene doped with hydrogen defects [28-30]. According to Lieb's theorem [31], this is expected to occur for bi-partite lattices when two impurities are on the same sublattice. Even though phosphorene has four sublattices (and hence, the theorem does not rigorously apply), the system may still realize similar physics. Unfortunately, our approach can only describe non-interacting/quadratic Hamiltonians and we cannot prove this conjecture. On the other hand, the dominant anti-ferromagnetism at all dopings and distances (more robust than in graphene [21]) indicates a route toward realizing dilute $2 \mathrm{D}$ anti-ferromagnetism with phosphorene.

Acknowledgements - The authors are grateful to the U.S. Department of Energy, Office of Basic Energy Sciences, for support under grant DE-SC0014407.

[1] R B Jacobs, Phosphorus at high temperatures and pressures, J. Chem. Phys. 59, 945 (1937). 
PAPERS IN PHYSICS, VOL. 9, ART. 090008 (2017) / A. Allerdt et al.

[2] L Pauling, M Simonetta, Bond orbitals and bond energy in elementary phosphorus, J. Chem. Phys. 20, 29 (1952).

[3] R R Hart, M B Robin, N A Kuebler, 3p orbitals, bent bonds, and the electronic spectrum of the $p 4$ molecule, J. Chem. Phys. 42, 3631 (1965).

[4] H Liu et al., Phosphorene: An unexplored $2 d$ semiconductor with a high hole, ACS Nano 8, 4033 (2014).

[5] A N Rudenko, M I Katsnelson, Quasiparticle band structure and tight-binding model for singleand bilayer black phosphorus, Phys. Rev. B 89, 201408 (2014).

[6] A S Rodin, A Carvalho, A H Castro Neto, Straininduced gap modification in black phosphorus, Phys. Rev. Lett. 112, 176801 (2014).

[7] L Li et al., Black phosphorus field-effect transistors, Nat. Nanotechnol. 9, 372 (2014).

[8] R W Keyes, The electrical properties of black phosphorus, Phys. Rev. 92, 580 (1953).

[9] X Ling, H Wang, S Huang, F Xia, M S Dresselhaus, The renaissance of black phosphorus, $\mathrm{P}$. Natl. Acad. Sci. USA 112, 4523 (2015).

[10] V Tran, R Soklaski, Y Liang, L Yang, Layercontrolled band gap and anisotropic excitons in few-layer black phosphorus, Phys. Rev. B 89, 235319 (2014).

[11] A Carvalho et al., Phosphorene: From theory to applications, Nat. Rev. Mater. 1, 16061 (2016).

[12] V V Kulish, O I Malyi, C Persson, P Wu, Adsorption of metal adatoms on single-layer phosphorene, Phys. Chem. Chem. Phys. 17, 992 (2015).

[13] P Rastogi, S Kumar, S Bhowmick, A Agarwal, Y S Chauhan, Effective doping of monolayer phosphorene by surface adsorption of atoms for electronic and spintronic applications, IETE J. Res. 63, 205 (2017).

[14] H-J Duan et al., Anisotropic RKKY interaction and modulation with mechanical strain in phosphorene, New J. Phys. 19, 103010 (2017).
[15] A C Hewson, The Kondo Problem to Heavy Fermions, Cambridge University Press, New York (1983).

[16] K Yosida, Magnetic properties of $\mathrm{Cu}-\mathrm{Mn}$ alloys, Phys. Rev. 106, 893 (1957).

[17] M A Ruderman, C Kittel, Indirect exchange coupling of nuclear magnetic moments by conduction electrons, Phys. Rev. 96, 99 (1954).

[18] T Kasuya, A theory of metallic ferro- and antiferromagnetism on Zener's model, Prog. Theor. Phys. 16, 45 (1956).

[19] A Allerdt, R Žitko, A E Feiguin, Nonperturbative effects and indirect exchange interaction between quantum impurities on metallic (111) surfaces, Phys. Rev. B 95, 235416 (2017).

[20] A Allerdt, C A Büsser, G B Martins, A E Feiguin, Kondo versus indirect exchange: Role of lattice and actual range of $R K K Y$ interactions in real materials, Phys. Rev. B 91, 085101 (2015).

[21] A Allerdt, A E Feiguin, S D Sarma, Competition between Kondo effect and RKKY physics in graphene magnetism, Phys. Rev. B 95, 104402 (2017).

[22] C A Büsser, G B Martins, A E Feiguin, Lanczos transformation for quantum impurity problems in d-dimensional lattices: Application to graphene nanoribbons, Phys. Rev. B 88, 245113 (2013).

[23] S R White, Density matrix formulation for quantum renormalization groups, Phys. Rev. Lett. 69, 2863 (1992).

[24] S R White, Density-matrix algorithms for quantum renormalization groups, Phys. Rev. B 48, 10345 (1993).

[25] A E Feiguin, The density matrix renormalization group method and its time-dependent variants, AIP Conf. Proc. 1419, 5 (2011).

[26] M Ezawa, Topological origin of quasi-flat edge band in phosphorene, New J. Phys. 16, 115004 (2014).

[27] B Zhou, B Zhou, X Zhou, G Zhou, Even-odd effect on the edge states for zigzag phosphorene nanoribbons under a perpendicular electric field, J. Phys. D: Appl. Phys. 50, 045106 (2017). 
PAPERS In Physics, VOL. 9, ART. 090008 (2017) / A. Allerdt et al.

[28] O V Zazyev, L Helm, Defect-induced magnetism in graphene, Phys. Rev. B 75, 125408 (2007).

[29] S Casolo, O M Løvvik, R Martinazzo, G F Tantardini, Understanding adsorption of hydrogen atoms on graphene, J. Chem. Phys. 130, 054704 (2009).
[30] H González-Herrero et al., Atomic-scale control of graphene magnetism by using hydrogen atoms, Science 352, 437 (2016).

[31] E H Lieb, Two theorems on the Hubbard model, Phys. Rev. Lett. 62, 1201 (1989). 